\title{
Revisión del estado del arte de las prácticas de cuidado infantil|2
}

\section{Luz Ibáñez Navarro}

Magíster en Desarrollo Social. Universidad de la Costa Universidad del Norte, Colombia Correo electrónico: libanez@cuc.edu.co Imibanez@uninorte.edu.co

\section{Camilo Madariaga Orozco}

PhD. En Educación. Universidad del Norte, Colombia.

\section{Resumen}

El presente trabajo tiene como objetivo desarrollar una revisión bibliográfica relacionada con la temática de prácticas de cuidado infantil con el propósito de construir un análisis a partir de los documentos revisados. La importancia de las prácticas de cuidado y el impacto de los patrones culturales sobre estas representan los ejes fundamentales del análisis desarrollado. Los documentos revisados muestran cómo la familia representa un escenario determinante en la potencialización de las distintas habilidades de los niños a través de la ejecución de las prácticas de cuidado.

\section{Palabras clave}

Cuidado del niño, infancia, prácticas de cuidado, desarrollo infantil.

2 Para citar este artículo: Ibáñez, I. \& Madariaga, C. (2020). Revisión del estado del arte de las prácticas de cuidado infantil. Informes Psicológicos, 20(2), pp. 23-40 http://dx.doi.org/10.18566/infpsic.v20n2a2 


\title{
Review of the state of the art of child care practices
}

\begin{abstract}
The objective of this work is to develop a bibliographic review related to the topic of child care practices with the purpose of constructing an analysis from the documents reviewed. The importance of care practices and the impact of cultural patterns on those represent the fundamental axes of the analysis. The reviewed documents show how the family represents a determining scenario in the empowerment of the different abilities of children through the execution of care practices.
\end{abstract}

Keywords

Child care, childhood, care practices, child development.

\section{Revisão do estado da arte das práticas de cuidado infantil}

\section{Resumo}

0 objetivo deste trabalho é desenvolver uma revisão bibliográfica relacionada com a temática das práticas de cuidado infantil, com o objetivo de construir uma análise a partir dos documentos revisados. A importância das práticas de cuidado e 0 impacto dos padrões culturais sobre elas representam os eixos fundamentais da análise desenvolvida. Os documentos revisados mostram como a família representa um cenário determinante na promoção das diferentes habilidades das crianças por meio da execução de práticas de cuidado.

Palavras chave

Cuidado infantil, infância, práticas de cuidado, desenvolvimento infantil. 


\section{ntroducción}

La familia cumple un papel determinante sobre el desarrollo humano dentro de la sociedad debido a que permite la construcción constante de escenarios que le posibilitan al niño desarrollar sus potencialidades y habilidades (Moliner, 2005). Sobre esto, Muñoz (2005) puntualiza que la familia representa el "contexto más deseable de crianza y educación de niños y adolescentes, ya que es quien mejor puede promover su desarrollo personal, social e intelectual y, además, el que habitualmente puede protegerlos mejor de diversas situaciones de riesgo" (p. 148.)

En este sentido, la familia tiene como una de sus funciones, proporcionar a los niños la oportunidad de fortalecer las distintas dimensiones de su desarrollo a través de una estimulación cognitiva adecuada, la enseñanza de valores, principios y conductas favorables (Domenech, Donovick \& Crowley, 2009; Rodrigo, Martín, Cabrera \& Máiquez, 2009; Valdivia, 2008) y la creación de un ambiente agradable, el cual, de acuerdo a Romagnoli y Cortese (2016) favorece el aprendizaje en los niños, lo que a su vez crea estados emocionales que facilitan el aprovechamiento de oportunidades que se les presentan dentro de estos mismos ambientes. En concordancia con esto, Gallego (2012) afirma que "la familia es el primer grupo humano en el que cada hombre y cada mujer tienen experiencias altamente significativas" (p. 67), lo que se soporta con la premisa de que una de las funciones de la familia es estimular a los niños para que desarrollen la capacidad de relacionarse de forma competente con su entorno físico y social (Muñoz, 2015). Así entonces, la familia se constituye como una unidad social, un espacio que puede cumplir una función determinante como agente socializador en la infancia de los hijos.

El cuidado y la crianza de los niños representan la función principal de los padres. Ante esto, Jorge y González (2017) destacan que "los padres son los principales gestores para el desarrollo de los hijos y son los pioneros en practicar los estilos de crianza, que influyen en los procesos de socialización" (p. 41). En todo caso, para esto no existe un manual que pueda utilizarse de forma universal debido a que las prácticas de cuidado se tornan susceptibles a la influencia de la cultura, es decir, los modos de vida, costumbres, conocimientos de los distintos grupos sociales. Sin embargo, actualmente existe un amplio marco científico que propone que determinadas prácticas de cuidado pueden presentar resultados más adecuados que otras, dejando éstas una huella imborrable que genera que, incluso en la adultez, el ser humano esté siempre vinculado a los cuidados bajo los que se desarrolló y a la crianza que recibió (Rojas, 2015).

En este sentido, es importante construir un trabajo que tenga en cuenta las diversas investigaciones que se han desarrollado en el marco de la temática de las prácticas de cuidado infantil. Así entonces, el presente artículo tiene como propósito realizar una revisión bibliográfica asociada a las prácticas de cuidado infantil, teniendo como foco de atención la importancia de dichas prácticas sobre el desarrollo infantil y el impacto que tiene la cultura sobre las mismas. Lo anterior, dada la exposición a la cultura de los niños 
desde su nacimiento, abarcando sus formas de dormir, prácticas alimenticias, la formación de su sistema de valores, sus interacciones interpersonales, entre otros aspectos que influyen, por ende, en su desarrollo (Greenfield \& Suzuki, s.f.).

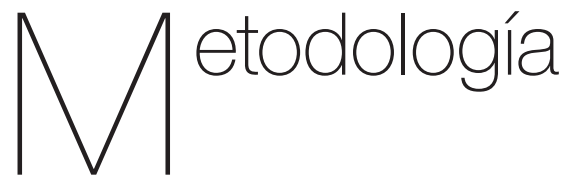

Como se mencionó, el propósito del presente artículo es construir un análisis a partir de una revisión de literatura científica asociada a las prácticas de cuidado para atender a dos cuestiones: la importancia de dichas prácticas sobre el desarrollo infantil y el impacto que tiene la cultura sobre las mismas.

Para cumplir con dicho objetivo se llevó a cabo una revisión basada en la búsqueda de literatura a partir de bases de datos indexadas (Scopus, Science Direct, Web of Science, Scielo y Redalyc). Los criterios de búsqueda se basaron en la revisión de 60 documentos científicos en idiomas español e inglés orientados al desarrollo cultural de las prácticas de cuidado y/o crianza en distintos contextos y su impacto sobre el desarrollo de los niños, documentos que en su mayoría se clasifican como artículos científicos, representando solo una minoría algunos libros y documentos académicos. Los descriptores utilizados en la búsqueda fueron: Prácticas de cuidado, cuidado infantil, desarrollo infantil y cultura.

Los principales autores revisados fueron Aguirre y Gallego (2002; 2012) en referencia al concepto de Práctica de Cuidado, Amar (2004; 2014) en torno al desarrollo infantil y Tenorio (1999) y Greenfield y Suzuki (s.f.) en relación al concepto de cultura.

De este modo, se revisaron estudios a partir de 1999 teniendo en cuenta libros y artículos con un amplio aporte a la conceptualización de la temática; no obstante, la mayor parte de las investigaciones revisadas se encuentran condensadas entre los últimos 10 años. Finalmente, se pasó a analizar detenidamente los artículos revisados a partir de las categorías de análisis: Impacto sobre el desarrollo infantil e influencia cultural, para, de esta forma, desarrollar aportes de investigación dentro del campo temático.

Teniendo en cuenta lo anterior, a continuación, se presenta la integración de las principales ideas derivadas de la revisión bibliográfica realizada. A partir de esto, se inicia con la relación entre prácticas de cuidado y su importancia en el desarrollo infantil y se finaliza con el impacto de la cultura sobre estas.

\section{Prácticas de cuidado y desarrollo infantil}

El desarrollo ha sido caracterizado mediante distintos puntos de vista que van desde lo económico hasta la social. Actualmente la UNICEF, citado en Amar, Abello y Tirado (2004), subraya que el desarrollo se define como una perspectiva que concibe al ser humano como sujeto de los aspectos tanto económicos y sociales del contexto, atendiendo a sus derechos como persona y, más específicamente, "los derechos del niño como 
objetivos y metas culturales que permiten fomentar la construcción de conocimientos útiles a la conservación y el desarrollo integral como meta principal de todos los programas en beneficio de las personas" (Amar et al., 2004, p. 2).

A partir de lo anterior, el desarrollo del ser humano se relaciona con un enfoque integral que no sólo tiene en cuenta las distintas dimensiones y realidades del sujeto, sino que, además, atiende a los factores externos que influyen sobre él, por ende, condicionan su existencia. En este sentido, Amar et al. (2004) puntualizan que, según el enfoque holístico, enfoque que desarrolla análisis de los fenómenos desde su totalidad y de una forma completa, el desarrollo puede ser definido como el proceso mediante el cual las características físicas y biológicas del individuo transcienden hacia niveles sociales y culturales, destacando el papel activo de éste y el carácter constructivo del desarrollo a través de la constante interacción con los demás. En otras palabras, "sería la realización del potencial biológico, social y cultural de la persona" (Amar et al., 2004, p. 3).

El ser humano indudablemente necesita del otro para sobrevivir durante sus primeros años de vida ya que su existencia es prácticamente imposible sin la presencia de los cuidados físicos, e incluso afectivos, por parte de un cuidador (Gallego, 2012; Pulido, Castro, Peña \& Ariza, 2013). El papel de los padres de familia y demás cuidadores en la estimulación y potencialización de las dimensiones del desarrollo es importante y puede contribuir o dificultar el adecuado despliegue de éstas mediante las distintas acciones llevadas a cabo en el seno familiar. Sobre esto, Aguirre (2002) recalca que "las prácticas de crianza hacen parte de las relaciones familiares y en ellas se resalta el papel que juegan los padres en la formación de sus hijos" (p. 13).

Así entonces, las prácticas de cuidado o pautas de crianza pueden referirse a muchos aspectos relacionados con el quehacer de los padres de familia y/o cuidadores primarios de los niños. En este sentido, Cuervo (2010) afirma que las prácticas de cuidado se destacan como aquellas "acciones llevadas a cabo por los padres y personas responsables del cuidado del niño/a para dar respuesta cotidianamente a sus necesidades" ( $p$. 115).

Por su parte, Aguirre (2002) enfatiza que las prácticas de cuidado poseen cuatro características fundamentales. La primera de ellas se centra en que dichas prácticas se constituyen como una relación de poder donde se evidencia la clara intención de los cuidadores de cumplir su función orientadora con los niños.

La segunda característica de las prácticas de cuidado subraya que éstas no se manifiestan en una sola vía de influencia, sino que la relación existente entre cuidador y niño refleja una mutualidad que se percibe en el hecho de que los niños también pueden ejercer control sobre el comportamiento de sus padres y/o cuidadores en general.

La tercera característica de las prácticas de crianza, según Aguirre (2002), es que éstas en realidad representan un proceso, lo que indica que "son un conjunto de acciones concatenadas, que cuenta un inicio y que se va desenvolviendo conforme pasa el tiempo" (Aguirre, 2002, p. 14). Esto también implica 
que las prácticas de cuidado no deben ser concebidas como conductas estáticas y repetitivas ejercidas por los padres y/o cuidadores.

Una cuarta característica de las prácticas de crianza destaca que son aprendidas debido a que son ejercidas por parte de los cuidadores tomando a otros padres o a los propios como referencia conductual. Además de esto, la cultura en la que se desenvuelven los cuidadores también juega un papel muy importante ya que las creencias y representaciones sociales dictaminan e influyen las acciones para con los niños.

Asimismo, este constructo puede definirse como "el conjunto de acciones que los sujetos adultos de una cultura realizan, para orientar hacia determinados niveles y en direcciones específicas, el desarrollo de los sujetos, igualmente activos, pero más pequeños, del grupo" (OEA, 1997, citado en Gómez, Gómez \& Hurtado, 2006, p. 48).

Según Amar y Martínez (2014) el concepto de cuidado puede desglosarse mediante tres términos a explicar. El primero de estos es la práctica, definida por Aguirre (2002) como conductas que se orientan a garantizar la supervivencia del infante, beneficiando su crecimiento y desarrollo en términos físicos, psicológicos, sociales y emocionales. De acuerdo a Izzedin y Pachajoa (2009), las prácticas de cuidado "se ubican en contexto de las relaciones entre los miembros de la familia donde los padres juegan un papel importante en la educación de sus hijos" (p. 109).

El segundo término a considerar es el de pauta social que, según Amar y
Martínez (2014), se asocia con las costumbres culturales relacionadas con las prácticas de cuidado y que actúan como marco de referencia a la hora de ejercer las acciones de los padres sobre sus hijos. En este sentido, en las pautas predominan las representaciones sociales del niño, las cuales "condicionan la interpretación de los diferentes órdenes normativos, que pueden asumir formas bastante restrictivas o muy tolerantes, dándose entre estas una variedad, que depende de los rasgos culturales del grupo" (Aguirre, 2002, citado en Amar \& Martínez, 2014, p. 93).

Para finalizar, las creencias se constituyen como las explicaciones que otorgan los padres para sustentar la forma como orientan los comportamientos de sus hijos. Estas explicaciones son, por tanto, autorizadas por la sociedad y acorde a lo planteado en ésta (Amar \& Martínez, 2014). En torno a esto, Izzedin y Pachajoa (2009) señalan que las creencias se refieren al conocimiento sobre la forma en que debe ser criado un niño y a las distintas razones otorgadas por los padres acerca de la manera en que ejercen sus prácticas de cuidado.

En este orden de ideas, las distintas definiciones presentadas en torno a las prácticas de cuidado muestran que este constructo representa aquellas acciones llevadas a cabo por el personal encargado del cuidado de los niños, entiéndase padres, abuelos, vecinos y/o demás familiares; acciones que de una u otra forma tienen un impacto significativo sobre el desarrollo infantil y que, además, se encuentran influenciadas por el entorno cultural, análisis del cual surge la necesidad de desarrollar la presente revisión bibliográfica a partir de esas vías. 
Sobre esto, Varela, Chinchilla y Murad (2015) indican que "en general las creencias son culturales y se transmiten de generación en generación, y además sirven de soporte y brindan confianza en la interrelación padres/cuidadores- niñosniñas; parte de esas creencias aluden a cómo se deben cuidar los niños" ( $p$. 202). Lo anterior, deja entrever la forma en que práctica, pauta y creencia se interrelacionan entre sí, alimentándose mutuamente.

Por otra parte, Barkley (2013) puntualiza que existen prácticas de cuidado adecuadas e inadecuadas que pueden promover u obstaculizar la dinámica familiar respectivamente. Además, Vera, Grubits y Rodríguez (2007) señalan que las prácticas de cuidado del niño hacen parte de "un constructo más psicológico que se incluye dentro de estilo de crianza y que implica el estudio del microambiente familiar y la manera en la cual favorecen u obstaculizan procesos de salud, alimentación y desarrollo" (p. 51).

Así entonces, se encuentran por un lado las pautas de crianza negativas que incluyen elementos como las discusiones presentadas dentro del entorno familiar, la negación del afecto y manifestaciones de agresividad, entre otras, las cuales, a su vez, pueden generar comportamientos agresivos en los mismos niños, quienes continúan la tendencia observada en sus padres. Sobre esto, Cabrera, González y Guevara (2012) señalan que la familia juega un papel relevante tanto en la prevención como en la promoción de la conducta de agresión infantil, la cual, de acuerdo a los autores, puede anteceder el desarrollo de comportamientos antisociales durante la adultez.
Por otro lado, se encuentran las pautas de crianza positivas que actúan como facilitadoras del desarrollo infantil, entre las que se destacan diferentes acciones tales como las expresiones de afecto, la proporción de instrucciones claras y coherentes a los niños, la comprensión y el buen ejemplo en general (Barkley, 2013). En relación a esto, Builes, Múnera, Salazar y Schnitter (2006) indican que algunas prácticas de cuidado se constituyen como factores protectores (en este caso, las positivas) en torno a las relaciones desarrolladas por los niños dentro de la sociedad, caso contrario a aquellas prácticas de cuidado negativas basadas en el maltrato, las cuales se convierten en factores de riesgo para la primera infancia debido a la negación de afecto y promoción de comportamientos agresivos, entre otros aspectos.

Goodnow (1985, citado en López, Peña \& Rodríguez, 2008) menciona que las diversas acciones que ejercen los cuidadores y que son señaladas por estos como adecuadas y deseables para sus niños, se denominan estrategias de socialización, las cuales se manifiestan en la riqueza del vínculo afectivo entre ambos, el grado de comunicación y las distintas prácticas llevadas a cabo con el objetivo de ejercer autoridad en el infante.

Richaud (2005) desarrolló una investigación cuyo objetivo era determinar cómo los estilos parentales influyen sobre las estrategias de afrontamiento en los niños. Los resultados de tal estudio reflejan que aquellas familias que poseen un estilo democrático o asertivo en su parentalidad tienden a promover estrategias de afrontamiento adecuadas, adaptativas. Por su parte, las familias cuyas prácticas de cuidado y crianza se basan más 
en un estilo parental autoritario, tienden a promover la evitación de los problemas y la inseguridad en sus niños, fomentando así, estrategias de afrontamiento inadecuadas para ellos. Lo anterior, da cuenta de cómo las prácticas de cuidado ejercidas por los padres de familia tienen un impacto incluso en la forma como los niños comprenden el mundo y afrontan las situaciones problemáticas que se les puedan presentar.

Así, Henao, Ramírez y Ramírez (2007) indican que "la combinación de las costumbres y hábitos de crianza de los propios padres, la sensibilidad hacia las necesidades de su hijo, la aceptación de su individualidad y la del otro (...) son la base de las estrategias de socialización" (p. 236). De este modo, los padres de familia implementan sus distintas herramientas de regulación conductual en sus hijos y forman sus propios estilos educativos de crianza.

Los resultados de las investigaciones destacadas hasta el momento evidencian que las prácticas de cuidado representan un papel fundamental en el proceso de formación y desarrollo de los niños y niñas, en especial, de aquellos que se encuentran en sus primeros años de vida, debido a su alto grado de dependencia de sus cuidadores y/o adultos mayores a su alrededor y pertenecientes a su contexto. Lo anterior, se relaciona con lo señalado por Yárnoz (2006) en su revisión sobre el papel del padre dentro de la dinámica familiar. En este sentido, el autor subraya constantemente la importancia de los padres y/o cuidadores sobre el proceso de socialización de los niños y su papel fundamental como figuras de apego y modelos para las futuras relaciones personales de los infantes durante su vida.

En relación a esto, Torío, Peña y Rodríguez (2008) llevaron a cabo una investigación que tenía como objetivo estudiar las distintas prácticas de cuidado y los estilos de crianza de los cuidadores de niños y adolescentes, en asociación con el desarrollo de estos. Los resultados de esta revisión bibliográfica evidenciaron diversos estudios que reflejan la forma como las prácticas de cuidado y crianza relacionadas con la imposición de autoridad en los niños, presentan efectos adversos sobre el proceso de socialización de estos, afectando negativamente su independencia, confianza en sí mismo, creatividad, autoestima y conducta social. Por otro lado, aquellos padres cuyas prácticas de cuidado entran en la categoría de estilo permisivo, tienden a criar a los niños con un alto grado de dependencia y comportamientos antisociales, afectando así su madurez en su crecimiento y desarrollo e, incluso, su éxito a nivel personal (Torío et al., 2008).

Solís y Díaz (2007) llevaron a cabo una investigación que se centraba en conocer las diferentes prácticas de cuidado y pautas de crianza en madres y padres de niños menores de cinco años, desarrollando un análisis comparativo mediante las diferencias de género de los mismos. Los resultados manifestaron que las prácticas de cuidado de las madres se encuentran basadas más en la comunicación, a diferencia de los padres, quienes creen un poco más en la importancia del establecimiento de límites y autoridad en el marco de la crianza de sus hijos. Además, se encontró, según los datos, que las madres, gracias a su multiplicidad de roles, presentan mayor estrés durante la crianza 
de sus hijos, en muchas ocasiones debido a la falta de apoyo social por parte de su pareja, lo que se asimila a los resultados desarrollados por Mistry, Stevens, Sareen, De Vogli y Halfon (2007) en su investigación.

En esta misma línea, Cuervo (2010) realizó una revisión bibliográfica cuyo objetivo se centraba en la relación entre las pautas y estilos de crianza con el desarrollo socioafectivo durante la infancia. En su revisión se resaltó la importancia de los cuidadores como agentes facilitadores de la estimulación de conductas prosociales (Henao \& García, 2009; Zacarías, Aguilar \& Andrade, 2017), regulación emocional (Amaya, 2008) y prevención de dificultades asociadas a la salud mental (Díaz et al., 2006) tales como depresión (Mitchell, 2010), agresividad (Pichardo, Justicia \& Fernández, 2009), baja autoestima (Papalia, Wendkos \& Duskin, 2010) y ansiedad (Franco, Pérez \& de Dios, 2014).

En torno al cuidado físico del niño, Rodríguez (2014) desarrolló una investigación cuyo objetivo estaba centrado en la identificación de creencias y prácticas culturales sobre el cuidado infantil en el municipio de Chocontá, Cundinamarca, mediante entrevistas no estructuradas incluidas en un estudio de diseño cualitativo. Los resultados mostraron la presencia de prácticas de cuidado relacionadas con el cambio de pañal, el baño, la alimentación y la salud que deben ser modificadas en esta comunidad por el riesgo en que exponen a los niños.

En torno a esta misma dimensión física, Pelcastre et al. (2005) realizaron una investigación de tipo cualitativo mediante entrevistas, que tenía como finalidad caracterizar las prácticas de cuidado ejercidas por las parteras tradicionales en San Luis de Potosí, México. El estudio demostró que tales prácticas son comunes en las comunidades que carecen de servicios de salud. Así, también se mostró que las funciones de estas mujeres no están asociadas únicamente al parto, sino además a la nutrición de los niños, los cuidados prenatales y postnatales.

Sobre este mismo tema, Delgado, Calvache, del Cairo, Bedoya y Tabares (2006) llevaron a cabo un estudio de tipo cualitativo que tenía como finalidad identificar las prácticas de cuidado de las madres con sus niños recién nacidos en la Costa Pacífica Caucana de Colombia. La investigación, desarrollada mediante entrevistas semiestructuradas y grupos focales, mostró que las conductas de las madres apuntan a la lactancia del niño, el cuidado de su piel, su nutrición y a la atención a dificultades respiratorias y digestivas.

Bajo esta misma línea, Shloim, Edelson, Martin y Hetherington (2015) desarrollaron una revisión sistemática sobre las relaciones entre los estilos de crianza, las prácticas de cuidado asociadas a la alimentación y el peso de niños en primera infancia. Dicha revisión mostró asociaciones entre el estilo de crianza y el Índice de Masa Corporal en los niños. De este modo, se evidenció que las prácticas de cuidado vinculadas a una crianza indulgente y sin involucramiento por parte de los padres se relacionan con un riesgo de obesidad para los niños, mientras que los estilos de crianza con autoridad se asocian a un Índice de Masa Corporal saludable.

En relación a la dimensión socioemocional, se encuentran estudios como el realizado por Franco et al. (2014) cuyo 
objetivo se enfocaba en investigar la relación entre las prácticas de crianza de los padres y el desarrollo de síntomas de ansiedad y conductas disruptivas en niños de 3 a 6 años de edad. Los resultados, medidos y analizados en una forma cuantitativa, muestran que determinadas pautas de cuidado relacionadas con el nivel de apoyo brindado, la disciplina impartida o la autonomía otorgada influyen significativamente en la presencia o ausencia de comportamientos disruptivos y alteraciones emocionales en los niños de tales edades (Franco et al., 2014).

En torno a esta misma temática, Cprek, Williams, Asaolu, Alexander y Vanderpool (2015) llevaron a cabo un estudio cuyo objetivo se centraba en investigar la relación entre tres prácticas parentales positivas (leerle a los niños, hacerlos partícipes en la narración de cuentos y en cantos y comer juntos como una familia) y el desarrollo infantil asociado a las dimensiones sociales y conductuales. Los datos del estudio mostraron fuertes correlaciones entre cada una de las prácticas positivas y el desarrollo social y conductual del niño, lo que le permitió a los autores concluir que tales prácticas de cuidado por parte de los padres pueden reducir los riesgos de retraso en los niños para tales áreas.

Gallego (2012), por su parte, realizó un estudio que pretendía investigar la forma en que familias monoparentales femeninas experimentan sus prácticas de crianza en el marco de una metodología hermenéutica como herramienta para comprender la realidad de los participantes de dicho estudio. La investigación mostró que las percepciones que tienen las madres con respecto a la crianza de sus hijos están basadas en el cuidado y la educación de estos, destacando la importancia que tiene su constante presencia en el hogar a la hora de atender las necesidades de sus hijos a nivel físico, social y emocional.

De igual forma, estas madres indicaron que la coherencia durante la crianza representa un aspecto central a la hora de educar adecuadamente a sus hijos, debido a que, tal y como las participantes del estudio expresan, es fundamental ser consistentes entre lo que se le demanda a los niños y lo que se demuestra conductualmente en la cotidianeidad, señalando así que el ejemplo constituye la principal herramienta de transmisión de principios y valores en la educación (Gallego, 2012).

Asimismo, González y Estupiñán (2010) analizaron las prácticas de cuidado de madres adolescentes en el Municipio de Duitama, Boyacá, mediante una perspectiva comprensiva en el marco de la metodología cualitativa. El objetivo se logró en la medida en que los investigadores pudieron estudiar la forma como estas madres cumplen su figura autoritaria en sus niños pequeños a través de castigos, prohibiciones e introyección de la norma mediante verbalizaciones cuyo objetivo es la asimilación del respeto y el amor por parte de los niños.

Además de esto, el estudio muestra que las prácticas de cuidado relacionadas con la dimensión corporal del niño son, en general, delegadas a la abuela debido a que representa una figura de conocimiento y apoyo emocional con estas madres, quienes estimulan el sueño y la alimentación de sus niños a través de la creación de un entorno lúdico que facilita la generación de hábitos adecuados relacionados con las mencionadas 
dimensiones (González \& Estupiñán, 2010).

Por su parte, Alonso y Román (2005) desarrollaron una investigación cuyo objetivo se centraba en hallar la correlación existente entre las prácticas socializadoras de múltiples familias en distintos estratos socioeconómicos y la autoestima y autoconcepto de niños entre 3 y 5 años. Los resultados de dicho estudio manifiestan que aquellas familias que practican un estilo parental equilibrado/democrático, e incluso permisivo, tienden a criar niños con mayores grados de autoestima, a diferencia de las familias que ejercen un estilo parental de tipo autoritario.

En torno a esta misma dimensión, Isaza y Henao (2012) llevaron a cabo una investigación que tenía como objetivo determinar la influencia del clima sociofamiliar y los estilos de interacción parental sobre el desarrollo de habilidades sociales en niños y niñas. La investigación propone que los padres permisivos actúan como limitantes del desarrollo de sus niños en este nivel. Además de esto, se mostró que los padres autoritarios se constituyen como obstaculizadores del desempeño en sus hijos mientras que los padres con un estilo de crianza enmarcado dentro de la democracia, forman niños seguros, independientes y adaptados socialmente.

\section{Prácticas de cuidado y cultura}

La cultura, como sistema de creencias propias de un determinado contexto, permea las distintas conductas llevadas a cabo por parte de las personas pertenecientes a dicho contexto, lo cual no representa una excepción para las acciones ejercidas como cuidado infantil dentro de una sociedad (Austin, 2000; Vielma, 2003). De acuerdo a Keller et al. (2004), la parentalidad representa un mecanismo de transmisión cultural (Álvarez, Permberty, Blandón \& Grajales, 2012), es decir, de creencias, valores, normas y prácticas. De esta forma, y por ser una forma de transmisión, los significados relacionados con la crianza misma varían a lo largo del tiempo (Bocanegra, 2007).

En consecuencia, es importante destacar que, así como la crianza se alimenta de la cultura, entendida ésta como el conjunto de costumbres que caracterizan a un pueblo, asimismo la cultura se alimenta de la crianza en la medida en que esta última manifiesta y permite conocer los distintos patrones normativos y culturales propios de una determinada sociedad (Bornstein, 2012).

En relación a la importancia de la cultura sobre el ejercicio de las prácticas de cuidado, Noguera y Rodríguez (2008) puntualizan que el personal de salud asociado al cuidado infantil deben desarrollar su trabajo de forma congruente a nivel cultural, de tal forma que el punto de partida sea el saber profesional, pero que se busque comprender las prácticas de crianza ejercidas por las familias para establecer una comunicación asertiva sin que los cuidadores se sientan ofendidos, a su vez, permitiendo la permanencia de su legado cultural. Con respecto a esto, Varela et al. (2015) afirman que la crianza representa "un aspecto en la realidad, permite comprender las realidades socioculturales diversas, las representaciones simbólicas, las creencias, los patrones, los hábitos, las pautas, las normas y los sistemas o prácticas de crianza en 
los procesos formativos de los niños" ( $p$. 197).

En torno a la construcción de lo que implican las significaciones de crianza por parte de un cuidador, Peñaranda (2011) destaca que, si bien la cultura tiene un impacto sobre el desarrollo de dichos significados, la experiencia vital de la persona en particular también jugará un papel importante dentro de esa construcción. Así, se integran aspectos socio-ontológicos propios de las vivencias de los padres del niño y elementos histórico-culturales relacionados con la pertenencia a una determinada cultura.

Sobre esta influencia de la cultura en las prácticas de cuidado, Tenorio (1999) desarrolló una investigación que tenía como objetivo conocer la cultura detrás de la crianza en población afroamericana del Pacífico Colombiano. Los resultados de dicha investigación muestran que en la población estudiada (Taparal, Chocó), las prácticas de cuidado se desarrollan acorde a los modelos tradicionales. En este sentido, de acuerdo al artículo, en esta población la tendencia es que los niños sean llevados desde pequeños a colaborar con su padre en el trabajo que desempeñen y las niñas, por su parte, permanecen con la madre realizando determinados oficios de la casa.

En relación a esto, Buitrago, Cabrera y Guevara (2009) desarrollaron una investigación cualitativa cuya finalidad era comprender las representaciones sociales de castigo y género entre los adultos, en asociación con las medidas correctivas ejercidas por los cuidadores con sus niños y niñas, realizando a su vez una comparación entre distintos tipos de familia.
En general, los resultados reflejan una mayor presencia de correctivos y representaciones asociadas con el castigo en familias lideradas por madres cabeza de hogar, lo que, según el análisis de los autores, "termina evidenciando una ruptura del tradicional modelo patriarcal de la crianza y posibilitando así el desarrollo de las niñas y los niños más desde el desarrollo de sus competencias y no desde el rol esperado para hombres y mujeres" (Buitrago et al., 2009, p. 67).

Pulido et al. (2013), por su parte, se encargaron de estudiar las distintas representaciones sociales con respecto al castigo en un grupo de familias de la ciudad de Bogotá, en las que predomina el uso de esta práctica de cuidado. En el estudio se encontraron distintas concepciones en torno a éste, como el castigo como elemento formador del niño, en el que este tipo de correctivo se torna necesario en la medida en que actúa como herramienta de introyección de normas en el niño.

En segundo lugar, la creencia del castigo se presenta como medio ambivalente debido al reconocimiento de sus efectos, tanto positivos como negativos, dentro de la formación de los infantes. Por otro lado, se encuentra la concepción del castigo como maltratante debido a las consecuencias, tanto físicas como psicológicas, que pueden surgir en el niño, eliminándose cualquier efecto positivo dentro de esta concepción (Carreño \& Rey, 2010). Finalmente, el castigo como correctivo temido por los niños representa la última percepción reflejada en la investigación. En este último sentido, esta práctica de cuidado es concebida como un factor causante de terror en los niños (Pulido et al., 2013). 
Acorde con lo anterior, Triana, Ávila y Malagón (2010) llevaron a cabo un estudio en el departamento de Boyacá que consistía en la realización de un autodiagnóstico comunitario acerca de las conductas, actitudes y prácticas relacionadas con la crianza de niños y niñas menores de cinco años. El autodiagnóstico mostró que las prácticas de cuidado en el departamento se encuentran todavía fuertemente ligadas a la cultura machista y patriarcal presentes en la sociedad (Hernández, 2014). En este sentido, dentro de las dinámicas familiares, las responsabilidades vinculadas a la crianza y cuidado de los niños siguen siendo, de forma general, exclusivas de las madres, representando el padre una figura de autoridad y sustento económico.

Frente a esto, Rodríguez (2007) señala que, en general, la familia dinamiza el proceso de socialización del niño a partir de su forma de ver la vida, la cual, a su vez, se encuentra condicionada por aspectos sociales, económicos e históricos propios de la cultura a la que pertenecen.

Las prácticas de cuidado en ese sentido, representan un aspecto tan complejo en las relaciones dadas dentro de la dinámica familiar que se encuentran influenciadas no solo por la cultura en la que se desarrollen los cuidadores y niños, sino que, además, son susceptibles al impacto de los medios de comunicación y la tecnología. En este orden de ideas, Martínez, Pérez y Solano (2011) desarrollaron una investigación sobre los impactos de las nuevas tecnologías de la comunicación sobre la dinámica familiar y la forma en que éstas, a su vez, influencian las prácticas de cuidado en la medida en que representan un reto para los padres en el cumplimiento de su papel como cuidadores.

Dentro del análisis del artículo se plantea que los medios de comunicación y las nuevas tecnologías se constituyen como elementos sociales con impactos, tanto negativos como positivos, dentro de la crianza. En este sentido, se manifiesta que las consecuencias negativas se evidencian en el deterioro de la dinámica familiar gracias a la disminución del diálogo y del intercambio de emociones, sentimientos y vivencias entre los miembros de la familia, además de construir escenarios que fomentan el consumo excesivo y la violencia (Martínez et al., 2011).

No obstante, se destaca también que los medios de comunicación pueden constituirse como herramientas de aprendizaje en la medida en que actúan como modelos de imitación en los niños, quienes reproducen conductas asimiladas mediante tales medios (Posada, 2004). Así, el papel del cuidador se centraría en la elección de los contenidos que sus hijos visualizan y en su respectivo acompañamiento como reforzamiento de los aprendizajes (Martínez et al., 2011).

Como conclusión, Martínez et al. (2011) afirman que "todos los aspectos planteados evidencian el impacto que tienen las nuevas tecnologías de la comunicación y la información en las relaciones familiares" (Martínez et al., 2011, p. 117), dejando claro que los medios de comunicación y los nuevos contextos de desarrollo se asocian significativamente con las pautas de crianza y prácticas de cuidado presentes en los roles parentales, impactando también las relaciones que se configuran dentro de la dinámica familiar. 


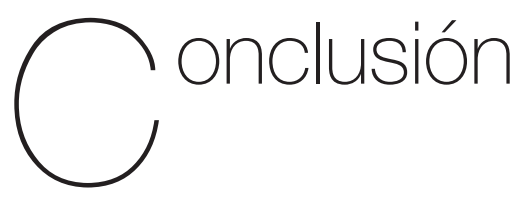

A partir de la revisión bibliográfica construida mediante la temática de prácticas de cuidado infantil, es posible desarrollar conclusiones basadas en la gran importancia que éstas tienen sobre el desarrollo y la forma como la cultura se constituye como un aspecto influyente dentro de la ejecución de las prácticas de cuidado llevadas a cabo en un contexto determinado.

Así, las prácticas de cuidado se constituyen como acciones que buscan potencializar las habilidades de los niños. Dichas acciones, llevadas a cabo generalmente por sus padres, son ejercidas con el fin de estimular las distintas capacidades de los niños de una forma integral. Atendiendo a esto, el papel de los padres de familia y demás cuidadores en la estimulación de las dimensiones del desarrollo infantil se torna fundamental en la medida en que puede contribuir o dificultar el adecuado despliegue de éstas mediante las distintas acciones llevadas a cabo en el seno familiar, por tal razón, es que éstas pueden representar factores tanto protectores como de riesgo para el desarrollo de los niños.

En este sentido, las prácticas de cuidado hacen parte de la dinámica familiar, manifiestan la relación entre sus miembros y, además, evidencian la importancia del papel que cumplen los cuidadores en la formación de los niños, en especial aquellos que se encuentran en infancia temprana, ya que es en esta etapa en donde se presentan importantes cambios para el desarrollo del niño.
Y es precisamente en estas prácticas de cuidado donde se ven reflejadas las funciones de la familia como sistema abierto y dinámico, debido a que la condición de fragilidad del niño al momento de su nacimiento hace indispensable su dependencia hacia un cuidador que le proporcione alimento, lo proteja de escenarios desfavorables, le otorgue abrigo; además, sea capaz de regular sus conductas a través de la observación, la identificación y la estimulación, así como de construir espacios propicios para la construcción de la autoestima, el autoconcepto y el adecuado manejo de las emociones.

De los argumentos anteriores se despliega la importancia de estudiar las prácticas de cuidado y desarrollar revisiones bibliográficas a partir de esta temática ya que su importancia sobre el cuidado infantil no se remite únicamente a aspectos físicos del infante, sino, además, presentan un impacto significativo sobre las áreas personales, sociales y emociones del desarrollo infantil.

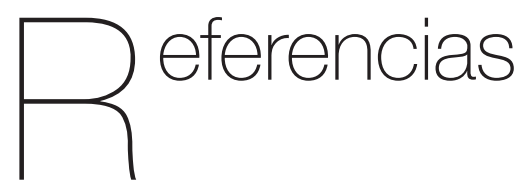

Aguirre, E. (2002). Prácticas de Crianza y Pobreza. Diálogos 2. Discusiones en la Psicología Contemporánea. Colección Debates en Psicología. Bogotá, D.C: Facultad de Ciencias Humanas, Universidad Nacional de Colombia.

Alonso, J. \& Román, J. (2005). Prácticas educativas familiares y autoestima. Psicothema, 17(1), 76-82. 
Álvarez, J., Pemberty, A., Blandón, A., \& Grajales, D. (2012). Otras prácticas de crianza en algunas culturas étnicas de Colombia: Un diálogo intercultural. El Ágora USB, 12(1), 89-102.

Amar, J., Abello, R., \& Tirado, D. (2004). Desarrollo infantil y construcción psicológica del mundo social. Barranquilla: Ediciones Uninorte.

Amar, J., \& Martínez, M. (2014). El ambiente imperativo: Un enfoque del desarrollo infantil. Barranquilla: Editorial Universidad del Norte.

Amaya, R. (2008). Parentalidad positiva: Educación emocional y en valores desde el ámbito familiar. Mesa Redonda: Educación Emocional y en Valores: Jornada sobre "Familia, escuela y sociedad: el reto de la convivencia". Madrid: Universidad de Oviedo.

Austin, T. (2000). Para comprender el concepto de Cultura. Revista UNAP Educación y Desarrollo, 1(1), 1-13.

Barkley, R. (2013). Defiant Children. A clinician's manual for assessment and parent training, third edition. New York: The Guilford Press.

Bocanegra, E. (2007). Las prácticas de crianza entre la colonia y la independencia de Colombia: los discursos que las enuncian y las hacen visibles. Revista Latinoamericana de Ciencias Sociales, Niñez y Juventud, 5(1), 1-21.

Bornstein, M. (2012). Cultural approaches to parenting. Parenting: Science and practice, 12(1), 212-221.

Builes, L., Múnera, C., Salazar, C. \& Schnitter, C. (2006). El encuentro de los Desencuentros:
Pautas de crianza presentes en las familias de niños menores de la calle institucionalizados. Informes Psicológicos, (8), 57-67.

Buitrago, M., Guevara, M., \& Cabrera, K. (2009). Las representaciones sociales de género y castigo y su incidencia en la corrección de los hijos. Revista Educación y Educadores, 12(3), 53-71.

Cabrera, V., González, M., \& Guevara, I. (2012). Estrés parental, trato rudo y monitoreo como factores asociados a la conducta agresiva. Universitas Psychologica, 11(1), 241-254.

Carreño, C., \& Rey, A. (2010). Reflexiones en torno a la comprensión del maltrato infantil. Universitas Psychologica, 9(3), 807-822.

Cprek, S., Williams, C., Asaolu, I., Alexander, L., \& Vanderpool, R. (2015). Three Positive Parenting Practices and Their Correlation with Risk of Childhood Developmental, Social, or Behavioral Delays: An Analysis of the National Survey of Children's Health. Maternal and Child Health Journal, 19(11), 2403-2411.

Cuervo, A. (2010). Pautas de crianza y desarrollo socioafectivo en la infancia. Revista Diversitas-Perspectivas en Psicología, 6(1), 111-121.

Delgado, M., Calvache, J., del Cairo, C., Bedoya, L., \& Tabares, R. (2006). Prácticas hogareñas en el cuidado de la madre y el recién nacido en la Costa Pacífica Caucana Antípoda. Revista de Antropología y Arqueología, (3), 227-254.

Díaz, D., Rodríguez, R., Blanco, A., Moreno, B., Gallardo, I. Valle, C., \& Dierendock, D. (2006). Adaptación española de las 
escalas de bienestar psicológico de Ryff. Psicothema, 18(3), 572-577.

Domenech, M., Donovick, M., \& Crowley, S. (2009). Estilos parentales en un contexto cultural: Observaciones del estilo parental protector en latinos de primera generación. Family Process, 48(2), 1-18.

Franco, N., Pérez, M., \& de Dios, M. (2014). Relación entre los estilos de crianza parental y el desarrollo de ansiedad y conductas disruptivas en niños de 3 a 6 años. Revista de Psicología Clínica con Niños y Adolescentes, 1(2), 149-156.

Gallego, T. (2012). Familias, infancias y crianza: Tejiendo Humanidad. Revista Virtual Universidad Católica del Norte, (35), 63-82.

Gallego, T. (2012). Prácticas de crianza de buen trato en familias monoparentales femeninas. Revista Virtual Universidad Católica del Norte, (37), 112-131.

Gómez, R., Gómez, Y., \& Hurtado, H. (2006). Interacción en el cuidado infantil. Dimensión psicológica y sociocultural. Medellín: La Carreta Editores E.U.

González, A., \& Estupiñán, M. (2010). Prácticas de crianza de madres adolescentes de la ciudad de Duitama, Colombia. Revista de Investigación y Educación en Enfermería, 28(3), 396-404.

Greenfield, P., \& Suzuki, L. (s.f.). Cultura y Desarrollo Humano: Implicaciones parentales, educativas, pediátricas y de salud mental. Instituto de PsicologíaGrupo Cultura y Desarrollo Humano.

Henao, G., Ramírez, C., \& Ramírez, L. (2007). Las prácticas educativas familiares como facilitadores del proceso de desarrollo en el niño y niña. Revista El Ágora, 7(2), 199-385.

Henao, G. Y., \& García, M. (2009) Interacción familiar y desarrollo emocional en niños y niñas. Revista Latinoamericana de Ciencias Sociales, Niñez y Juventud, 7(2), 785-802.

Hernández, J. (2014). La Empresa Familiar: como campo(s) de problemática(s) (Tesis de Pregrado). Universidad de la República, Montevideo. Uruguay.

Isaza, L., \& Henao, G. (2012). Influencia del clima sociofamiliar y estilos de interacción parental sobre el desarrollo de habilidades sociales en niños y niñas. Revista Persona, 15, 253-271.

Izzedin, R., \& Pachajoa, A. (2009). Pautas, Prácticas y Creencias acerca de Crianza... Ayer y Hoy. Liberabit: Revista de Psicología, 15(2), 109-115.

Jorge, E. \& González, C. (2017). Estilos de crianza parental: una revisión teórica. Informes Psicológicos, 17(2), 39-66.

Keller, H., Lohaus, A., Kuensemueller, P., Abels, M., Yovsi, R., Voelker, S. Jensen, H., Papaligoura, Z., Rosabal-Coto, M., Kulks, D., \& Mohite, P. (2004). The bio-culture of parenting: Evidence from five cultural communities. Parenting: Science and Practice, 4(1), 25-50.

López, S., Peña, J., \& Rodríguez, M. (2008). Estilos educativos parentales. Revisión Bibliográfica y Reformulación Teórica. Teoría de la Educación. Revista Interuniversitaria, 20, 151-178.

Martínez, M., Pérez, W., \& Solano, D. (2011). Impacto de los medios masivos de comunicación en la dinámica familiar. 
Revista Cultura, Educación y Sociedad, 2(1), 11-118.

Mistry, R., Stevens, G., Sareen, H., De Vogli, R., \& Halfon, N. (2007). Parenting-related stressors and self-reported mental health of mothers with young children. American Journal of Public Health, 97(7), 1261-1268.

Mitchell, B. (2010). Happiness in midlife parental roles: A contextual mixed methods analysis. Family Relations, 59(3), 326-339.

Moliner, R. (2005). La familia como espacio de socialización en la infancia. En Y. Corona \& N. del Rio (Coord.), Derechos de la Infancia. La infancia en riesgo (pp. 59-77). México: Editorial Universidad Autónoma Metropolitana-Xochimilco.

Muñoz, A. (2005). La familia como contexto de desarrollo infantil: Dimensiones de análisis relevantes para la intervención educativa y social. Revista Portularia, 5(2), 147-163.

Noguera, Y., \& Rodríguez, M.R. (2008). Aprendiendo a cuidar al recién nacido: un cuidado congruente con la cultura. Avances en Enfermería, 16(1), 103-111.

Papalia, D., Wendkos, S., \& Duskin, R. (2010). Desarrollo Humano. Undécima Edición. México, D. F: McGrawHill Interamericana Editores, S.A.

Pelcastre, B., Villegas, N., De León, V., Díaz, A., Ortega, D., Santillana, M., \& Mejía, J. (2005). Embarazo, parto y puerperio: Creencias y prácticas de parteras en San Luis Potosí, México. Revista Escolar de Enfermería, 39(4), 375-382.

Peñaranda, F. (2011). La crianza como complejo histórico, sociocultural y ontológico: una aproximación sobre educación en salud.
Revista Latinoamericana de Ciencias Sociales, Niñez y Juventud, 2(9), 945 - 956.

Pichardo, M., Justicia, F., \& Fernández, M. (2009). Prácticas de crianza y competencia social en niños de 3 a 5 años. Pensamiento Psicológico, 6(13), 37-48.

Posada, A. (2004). La crianza y los medios de comunicación social electrónicos. Curso continuo de actualización en Pediatría CCAP, 3(4), 23-45.

Pulido, S., Castro, J., Peña, M., \& Ariza, D. (2013). Pautas, creencias y prácticas de crianza relacionadas con el castigo y su transmisión generacional. Revista Latinoamericana de Ciencias Sociales, Niñez y Juventud, 11(1), 245-259.

Richaud, M. (2005). Estilos parentales y estrategias de afrontamiento en niños. Revista Latinoamericana de Psicología, 37(1), 47-58.

Rodrigo, M., Martín, J., Cabrera, E., \& Máiquez, M. (2009). Las competencias parentales en contextos de riesgo psicosocial. Psychosocial Intervention, 18(2), 113-120.

Rodríguez, A. (2007). Principales modelos de socialización familiar. Revista Foro de Educación, (9), 91-97.

Rodríguez, F. (2014). Prácticas y creencias culturales acerca del cuidado de niños menores de un año en un grupo de madres de Chocontá, Colombia. Revista Colombiana de Enfermería, 9(9), 77-87.

Rojas, M. (2015). Felicidad y estilos de crianza parental (Documento de Trabajo). Centro de Estudios Espinosa Yglesias, Puebla, México. 
Romagnoli, C. \& Cortese, I. (2016). ¿Cómo la familia influye en elaprendizajey rendimiento escolar? Recuperado de http://valoras. uc.cl/images/centro-recursos/familias/ ApoyoAlAprendizajeEnLaComunidad/ Fichas/Como-la-familia-influye-en-elaprendizaje-y-rendimiento.pdf

Shloim, N., Edelson, L., Martin, N., \& Hetherington, M. (2015). Parenting Styles, Feeding Styles, Feeding Practices, and Weight Status in 4-12 Year-Old Children: A Systematic Review of the Literature. Frontiers in Psychology, 6, 1-20.

Solís, P., \& Díaz, M. (2007). Relaciones entre creencias y prácticas de crianza de padres con niños pequeños. Revista Anales de Psicología, 23(2), 177-184.

Tenorio, M. (Mayo de 1999). Cultura y Crianza: Entre Tradición y Modernidad. Ponencia llevada a cabo en el IX Congreso Colombiano de Salud Mental y del Adolescente. Bogotá, Colombia.

Torío, S., Peña, J., \& Rodríguez, M. (2008). Estilos educativos parentales revisión bibliográfica y reformulación teórica. Revista Teoría de la Educación, 20, 151-178.

Triana, A., Ávila, L., \& Malagón, A. (2010). Patrones de crianza y cuidado de niños y niñas en Boyacá. Revista Latinoamericana de Ciencias Sociales, Niñez y Juventud, 8(2), 933-945.
Valdivia, S. (2008). La familia: concepto, cambios y nuevos modelos. La Revue du Redif, 1, 15-22.

Varela, S., Chinchilla, T., \& Murad, V. (2015). Prácticas de Crianza en niños y niñas menores de seis años en Colombia. Zona Próxima. Revista del Instituto de Estudios en Educación Universidad del Norte, (22), 193-215.

Vera, J., Grubits, S., \& Rodríguez, C. (2007). Estimulación y prácticas de crianza en infantes Terena del Brasil. Ra Ximhai, Revista de Sociedad, Cultura y Desarrollo Sustentable, 3(1), 49-81.

Vielma, J. (2003). Estilos de crianza, Estilos educativos y Socialización: ¿Fuentes de Bienestar Psicológico? Acción Pedagógica, 12(1), 48-55.

Yárnoz, S. (2006). ¿Seguimos descuidando a los padres? El papel del padre en la dinámica familiar y su influencia en el bienestar psíquico de sus componentes. Anales de Psicología, 22(2), 175-185.

Zacarías, X., Aguilar, E., \& Andrade, P. (2017). Efectos de las prácticas parentales en la empatía y la conducta prosocial de preadolescentes. Informes Psicológicos, 17(1), 71-86. http://dx.doi.org/10.18566/ infpsic.v17n1a04 